\title{
The Most Common Vitamin D Receptor Polymorphisms (ApaI, FokI, TaqI, BsmI, and BglI) in Children with Dental Caries: A Systematic Review and Meta-Analysis
}

\author{
Masoud Sadeghi ${ }^{1,2}{ }^{\oplus}$, Amin Golshah ${ }^{3}{ }^{\circ}$, Mostafa Godiny ${ }^{4}$, Roohollah Sharifi ${ }^{4}$, Atefeh Khavid ${ }^{5}$, \\ Nafiseh Nikkerdar ${ }^{5}$ and Santosh Kumar Tadakamadla ${ }^{6, * \text { (D) }}$ \\ 1 Medical Biology Research Center, Kermanshah University of Medical Sciences, Kermanshah 6714415185, Iran; \\ sadeghi_mbrc@yahoo.com \\ 2 Students Research Committee, Kermanshah University of Medical Sciences, Kermanshah 6715847141, Iran \\ 3 Department of Orthodontics, School of Dentistry, Kermanshah University of Medical Sciences, \\ Kermanshah 6713954658, Iran; amin.golshah@gmail.com \\ 4 Department of Endodontics, School of Dentistry, Kermanshah University of Medical Sciences, \\ Kermanshah 6713954658, Iran; mostafa_goodin@yahoo.com (M.G.); roholahsharifi@gmail.com (R.S.) \\ 5 Department of Oral and Maxillofacial Radiology, School of Dentistry, Kermanshah \\ University of Medical Sciences, Kermanshah 6713954658, Iran; atefehkhavid@gmail.com (A.K.); \\ n.nikkerdar@kums.ac.ir (N.N.) \\ 6 School of Medicine and Dentistry \& Menzies Health Institute Queensland, Griffith University, \\ Gold Coast 4214, Australia \\ check for \\ * Correspondence: santoshkumar.tadakamadla@griffithuni.edu.au
}

updates

Citation: Sadeghi, M.; Golshah, A. Godiny, M.; Sharifi, R.; Khavid, A.; Nikkerdar, N.; Tadakamadla, S.K. The Most Common Vitamin D Receptor Polymorphisms (ApaI, FokI, TaqI, $B s m I$, and $B g l I)$ in Children with Dental Caries: A Systematic Review and Meta-Analysis. Children 2021, 8 , 302. https://doi.org/10.3390/ children 8040302

Academic Editor: Tuan Nguyen

Received: 6 February 2021

Accepted: 12 April 2021

Published: 15 April 2021

Publisher's Note: MDPI stays neutral with regard to jurisdictional claims in published maps and institutional affiliations.

Copyright: (c) 2021 by the authors. Licensee MDPI, Basel, Switzerland. This article is an open access article distributed under the terms and conditions of the Creative Commons Attribution (CC BY) license (https:// creativecommons.org/licenses/by/ $4.0 /)$.
Abstract: Vitamin D participates in the calcification of enamel and dentin and the appropriate immune responses to oral microbial infections. We aimed to assess the association between the most common vitamin D receptor (VDR) polymorphisms (ApaI, FokI, TaqI, BsmI, and BglI) and the risk of dental caries in children. Methods: PubMed/MEDLINE, Cochrane Library, Web of Science, and Scopus databases were comprehensively searched until 19 January 2021. Meta-analysis with odds ratios as the effect estimate along with $95 \%$ confidence intervals and subgroup analysis were conducted using Review Manager 5.3 software. Publication bias and sensitivity analyses were conducted by Comprehensive Meta-Analysis, version 2.0 software. Results: Seventy-eight studies were retrieved from the databases, with nine studies included in the final analysis. Based on five genetic models, there was no association between ApaI (rs7975232), TaqI (rs731236), BsmI (rs1544410), FokI (rs2228570), and BglI (rs739837) polymorphisms and susceptibility to dental caries, except for the FokI (rs10735810) polymorphism. Conclusion: Among the VDR polymorphisms considered, an association was found between the FokI (rs10735810) polymorphism and the risk of dental caries, with a protective role of the $\mathrm{f}$ allele and ff genotype.

Keywords: dental caries; tooth decay; polymorphism; vitamin D; meta-analysis

\section{Introduction}

Dental caries is considered a complex and multifactorial disease as well as one of the most common diseases in industrialized and developing countries [1]. In the world, early childhood caries is considered to be the most common oral health problem in children [2] and is the most common childhood disease [3]. The age-standardized prevalence of dental caries in deciduous and permanent teeth was $7.8 \%$ and $29.4 \%$ and the number of prevalent cases was 532 and 2302 million in 2017, respectively [4]. In most developed countries, the prevalence of dental caries is declining sharply, while in developing countries, it is increasing [1]. Several genes such as genes included in enamel development, immune response, and saliva function can be associated with susceptibility to caries [5]. A genomewide meta-analysis [6] showed that consideration of the environment and aggregate genetic 
effects is more significant than specific genetic variants. A genome-wide association scan [7] reported that several genomic regions showed suggestive evidence for association with dental caries. The heritability of dental caries varies between 40 and 60\% [8-10]. Vitamin D is a fat-soluble steroid that is essential for maintaining the body's mineral balance [11], and it plays an important role in the calcification of enamel and dentin and the immune response to microbial infections of the mouth [12-15]. The function and biological activity of vitamin $\mathrm{D}$ are modulated by its interaction with the vitamin $\mathrm{D}$ receptor $(V D R)$ protein [16], and the activity of the $V D R$ protein is affected by polymorphisms of the $V D R$ gene [17]. More than 200 polymorphisms of the $V D R$ gene have been reported [18,19]. The VDR gene was found to impact the activity of a major metabolite of vitamin $D$, which participates in the formation of tooth enamel $[18,20]$, which demonstrates its potential implication for dental caries risk [21-23]. The most common functional VDR polymorphisms found to be potentially involved in oral and systemic conditions are BsmI, FokI, TaqI, BglI, and ApaI [24]. BsmI, TaqI, and ApaI polymorphisms were found to influence VDR protein structure, with FokI also influencing the transcriptional activity translation [25]. The aim of this meta-analysis is to evaluate the association between these VDR polymorphisms (ApaI, FokI, TaqI, BsmI, and BglI) and susceptibility to dental caries in children.

\section{Materials and Methods}

This systematic review was performed according to the Preferred Reporting Items for Systematic Reviews and Meta-Analyses (PRISMA) protocols [26].

\subsection{Data Sources and Literature Search}

Searches in PubMed/MEDLINE, Cochrane Library, Web of Science, and Scopus databases were comprehensively performed until January 19, 2021, without any restrictions. The search strategies for each database are shown in Table 1 The titles and abstracts were checked by two authors (M.S. and S.K.T.) and any disagreement was resolved by consensus with a third author (A.G.). We also checked the references of all included studies to ensure no study was missed.

Table 1. Search strategies.

\begin{tabular}{|c|c|}
\hline Database & Search \\
\hline Cochrane Library & 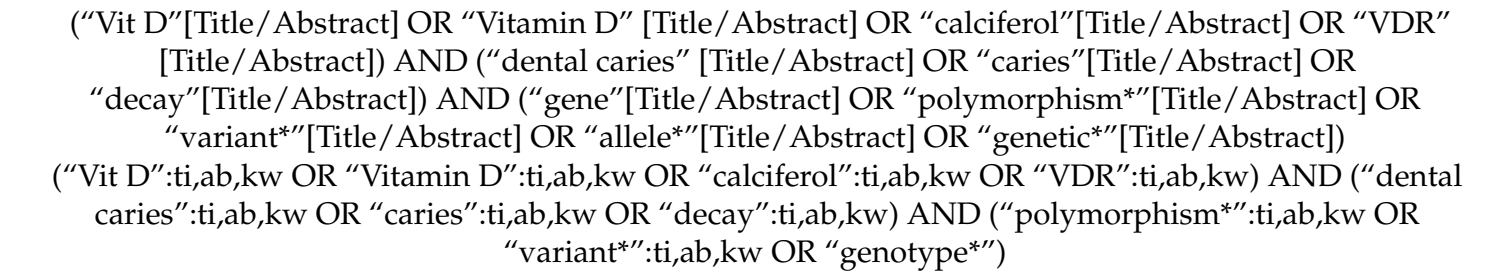 \\
\hline Web of Science & 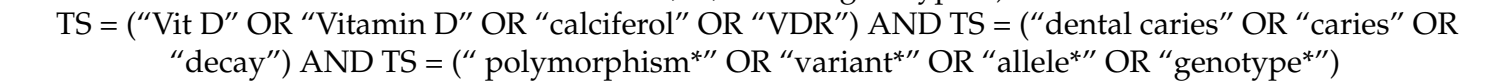 \\
\hline Scopus & 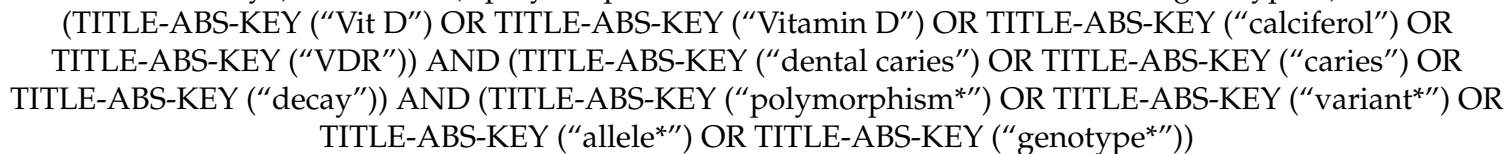 \\
\hline
\end{tabular}

\subsection{Eligibility Criteria and Study Selection}

The inclusion criteria were: (1) case-control studies focusing on the association between VDR polymorphisms and the risk of dental caries; (2) studies reporting VDR polymorphisms (ApaI (rs7975232), FokI (rs10735810), TaqI (rs731236), BsmI (rs1544410), FokI ( rs2228570), and BglI (rs739837)) in children (age < 18 years); (3) dental caries confirmed by clinical examinations; (4) studies reporting the frequencies of alleles or genotypes; and (5) a control group with no tooth decay. Reviews, conference papers, and studies with no control group or those among adults or reporting other polymorphisms of $V D R$ were 
excluded. The data from published studies were retrieved independently by two authors (M.S. and R.S.) to retrieve the necessary information. In case of discrepancies between the data extracted by the two authors, a duplicate data extraction was performed by a third author (M.G.).

\subsection{Quality Assessment}

Three reviewers (M.S., A.K., and N.N.) independently assessed the quality of the selected studies by scoring them according to Table 2 . We developed a quality assessment tool specifically for this study, which consisted of 7 criteria. The range of scores varies from 0 to 11 , with higher scores indicating better study quality.

Table 2. Criteria for quality assessment.

\begin{tabular}{|c|c|}
\hline Criteria & Score \\
\hline \multicolumn{2}{|l|}{ 1. Representativeness of cases } \\
\hline Consecutive/randomly selected from case population with clearly defined sampling frame & 2 \\
\hline $\begin{array}{c}\text { Consecutive/randomly selected from case population without clearly defined sampling frame or with extensive } \\
\text { inclusion/exclusion criteria }\end{array}$ & 1 \\
\hline Not described & 0 \\
\hline 2. Source of controls & \\
\hline Population- or community-based & 2 \\
\hline Hospital-based & 1 \\
\hline Not described & 0 \\
\hline \multicolumn{2}{|l|}{ 3. Ascertainment of dental caries } \\
\hline Clinical examination & 2 \\
\hline Diagnosis of caries by patient medical record & 1 \\
\hline Not described & 0 \\
\hline \multicolumn{2}{|l|}{ 4. Sample size } \\
\hline$>1000$ & 2 \\
\hline $200-1000$ & 1 \\
\hline$<200$ & 0 \\
\hline \multicolumn{2}{|l|}{ 5. Age and sex were matched between cases and controls } \\
\hline Yes & 1 \\
\hline No/Not described & 0 \\
\hline 6. Quality control of genotyping methods & \\
\hline Repetition of partial/total tested samples & 1 \\
\hline Not described & 0 \\
\hline 7. Hardy-Weinberg equilibrium in control subjects & \\
\hline Hardy-Weinberg equilibrium & 1 \\
\hline Hardy-Weinberg disequilibrium & 0 \\
\hline
\end{tabular}

\subsection{Statistical Analysis}

The association between polymorphisms and dental caries susceptibility was calculated by odds ratios (ORs) with 95\% confidence intervals (CIs) based on five genetic models (allele, homozygote, heterozygote, recessive, and dominant models). To calculate heterogeneity, a chi-square-based $\mathrm{Q}$ test and the $\mathrm{I}^{2}$ statistic were used $[27,28]$. A $p$-value of $>0.10$ and $\mathrm{I}^{2}<50 \%$ indicated that there was no heterogeneity between the studies. However, considering the diversity in the effect sizes and populations between the studies, we used a random effects model in all analyses. Subgroup analysis (based on ethnicity and genotyping method) and sensitivity analysis ("one study removed" and "cumulative analysis") were applied to find the effect of subgroups on the overall results and the stability of results, respectively. Funnel plots were used to determine publication bias. The $p$-value of (two-sided) $<0.05$ was considered significant, but the size of the effect was also taken into consideration to determine the association between the polymorphism and dental caries. The forest plots and subgroup analysis were conducted by Review Manager 5.3 (RevMan 5.3) software, while publication bias and sensitivity analyses were performed using Comprehensive Meta-Analysis version 2.0 (CMA 2.0) software. The polymorphisms 
(ApaI (rs7975232), FokI (rs10735810), TaqI (rs731236), BsmI (rs1544410), FokI (rs2228570), and BglI (rs739837)) were demonstrated to not be in strong linkage disequilibrium (LD) with each other $\left(\mathrm{r}^{2}<1\right)$ using the LDlink online tool (https:/ / ldlink.nci.nih.gov) (accessed on 6 November 2020) [29], and therefore all polymorphisms were included in the present meta-analysis.

\section{Results}

\subsection{Study Selection}

Seventy-eight studies were retrieved from the databases (Figure 1). After removing and excluding duplicate and irrelevant records, 14 full texts were evaluated for eligibility. Then, five full-text articles were excluded for different reasons: one article was a systematic review, one article had no control group, one article reported other $V D R$ polymorphisms, and two articles reported $V D R$ polymorphisms in adults. At last, nine studies were included in the qualitative and quantitative analysis.

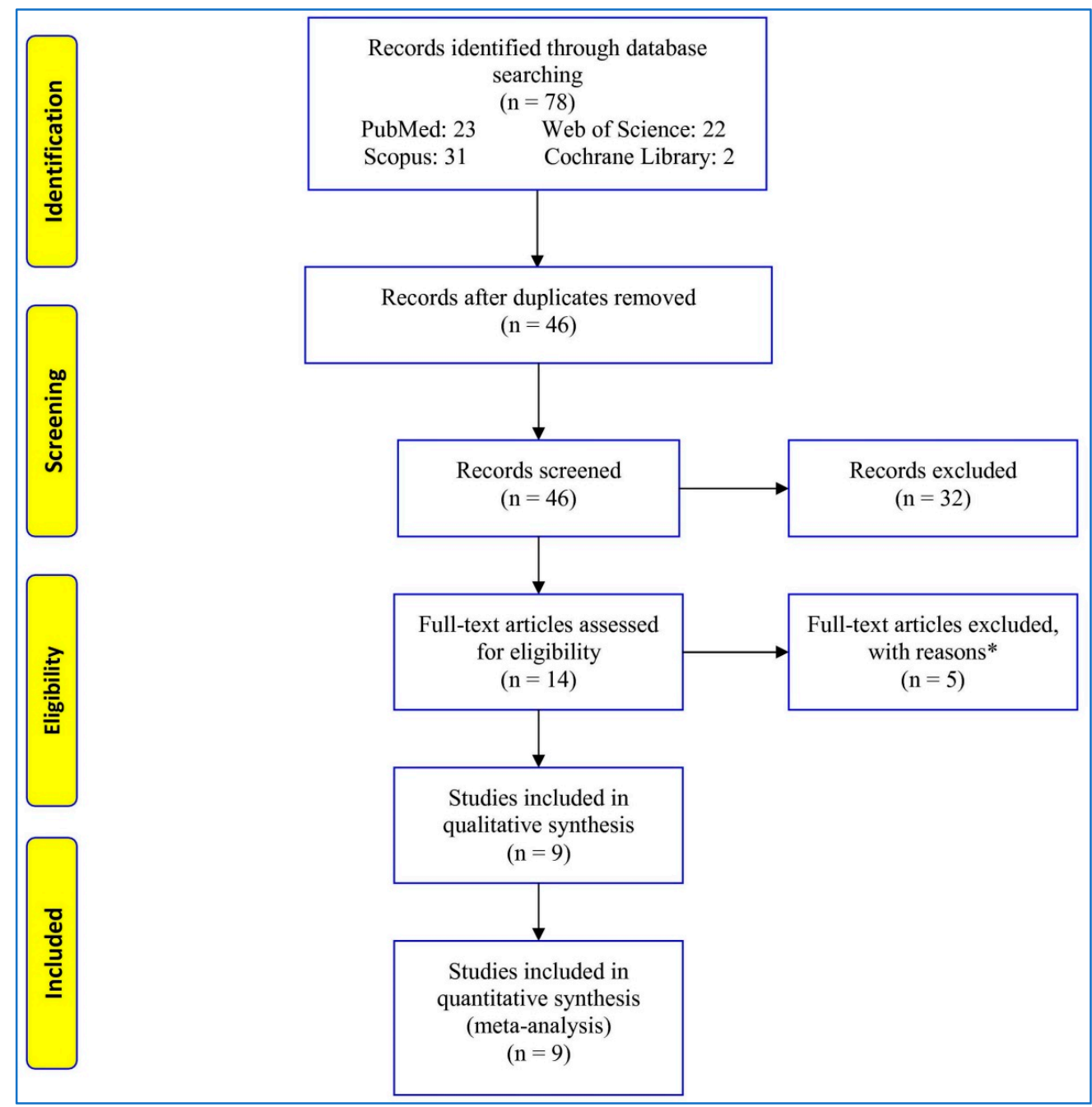

Figure 1. Flowchart of the study selection. * One article was a systematic review. One article had no control group. One article reported other vitamin D receptor $(V D R)$ polymorphisms. Two articles reported $V D R$ polymorphisms in adults.

\subsection{Quality Assessment}

The seven criteria used for quality assessment are shown in Table 2. The maximum possible score was 11 , while the minimum was 0 . 


\subsection{Characteristics of Studies}

Table 3 shows the characteristics of nine studies included in the meta-analysis [21,30-37]. Out of nine studies, three each were reported from China [21,36,37] and Brazil [31,33,35], and one each from Turkey [32], Czech Republic [34], and India [30]. There were three studies each on Caucasian, Asian, and mixed ethnic participants. The source of the control was population-based/school-based in all studies.

Table 3. Background characteristics of studies included in the meta-analysis.

\begin{tabular}{|c|c|c|c|c|c|}
\hline $\begin{array}{l}\text { First Author, } \\
\text { Publication Year }\end{array}$ & Country & Ethnicity & Source of Control & Genotyping Method & Quality Score \\
\hline Cogulu, 2016 [32] & Turkey & Caucasian & Population-based & PCR-RFLP & 7 \\
\hline Holla, 2017 [34] & Czech Republic & Caucasian & Population-based & TaqMan & 9 \\
\hline Kong, 2017 [21] & China & Asian & School-based & PCR & 8 \\
\hline $\mathrm{Yu}, 2017$ [37] & China & Asian & School-based & PCR-RFLP & 10 \\
\hline Qin, 2019 [36] & China & Asian & Population-based & TaqMan & 10 \\
\hline Aribam, 2020 [30] & India & Caucasian & Population-based & PCR & 9 \\
\hline Barbosa, 2020 [31] & Brazil & Mixed & School-based & Real-Time PCR & 8 \\
\hline Fatturi, 2020 [33] & Brazil & Mixed & School-based & Real-Time PCR & 10 \\
\hline Madalena, 2020 [35] & Brazil & Mixed & School-based & Real-Time PCR & 9 \\
\hline
\end{tabular}

Abbreviations: PCR, polymerase chain reaction; RFLP, restriction fragment length polymorphism.

The prevalence of alleles and genotypes of six polymorphisms is shown in Table 4. In addition, the $p$-value of the Hardy-Weinberg equilibrium (HWE) for controls is reported.

Table 4. Prevalence of alleles and genotypes of the polymorphisms in cases and controls.

\begin{tabular}{|c|c|c|c|c|c|c|c|c|c|c|c|c|c|c|}
\hline \multirow[t]{2}{*}{$\begin{array}{c}\text { First Author, } \\
\text { Publication Year }\end{array}$} & \multirow[t]{2}{*}{ Groups $(N)$} & \multicolumn{3}{|c|}{$\begin{array}{c}\text { ApaI } \\
(\text { rs7975232) }\end{array}$} & \multicolumn{3}{|c|}{$\begin{array}{c}\text { FokI } \\
(\text { rs10735810) }\end{array}$} & \multicolumn{3}{|c|}{ TaqI (rs731236) } & \multicolumn{3}{|c|}{ BsmI (rs1544410) } & \multirow[t]{2}{*}{$p$-Value of HWE } \\
\hline & & AA & $\mathrm{Aa}$ & aa & $\mathrm{FF}$ & $\mathrm{Ff}$ & $\mathrm{ff}$ & $\mathrm{TT}$ & $\mathrm{Tt}$ & $\mathrm{Tt}$ & $\mathrm{BB}$ & $\mathrm{Bb}$ & $\mathrm{bb}$ & \\
\hline \multirow{2}{*}{ Cogulu, 2016 [32] } & Case (112) & - & - & - & - & - & - & 35 & 46 & 31 & - & - & - & \multirow{2}{*}{0.132} \\
\hline & Control (38) & - & - & - & - & - & - & 15 & 14 & 9 & - & - & - & \\
\hline \multirow{2}{*}{ Holla, 2017 [34] } & Case (235) & - & - & - & - & - & - & 95 & 110 & 30 & - & - & - & \multirow{2}{*}{0.037} \\
\hline & Control (153) & - & - & - & - & - & - & 51 & 85 & 17 & - & - & - & \\
\hline \multirow{2}{*}{ Kong, 2017 [21] } & Case (249) & 44 & 87 & 118 & 69 & 132 & 48 & 230 & 19 & 0 & 0 & 152 & 97 & \multirow{2}{*}{$\begin{array}{c}0.011,0.662 \\
0.615, \text { and }<0.001\end{array}$} \\
\hline & Control (131) & 18 & 43 & 70 & 34 & 63 & 34 & 120 & 11 & 0 & 0 & 60 & 71 & \\
\hline \multirow{2}{*}{ Yu, 2017 [37] } & Case (200) & 33 & 85 & 82 & 86 & 96 & 18 & 171 & 29 & 0 & 0 & 36 & 164 & \multirow{4}{*}{$\begin{array}{c}0.210,0.057 \\
0.097, \text { and } 0.399 \\
0.895,0.764 \\
<0.001, \text { and } 0.909\end{array}$} \\
\hline & Control (200) & 24 & 79 & 97 & 65 & 86 & 49 & 158 & 42 & 0 & 0 & 31 & 169 & \\
\hline \multirow{2}{*}{ Qin, 2019 [36] } & Case (304) & 17 & 129 & 158 & 98 & 160 & 46 & 1 & 274 & 29 & 0 & 28 & 276 & \\
\hline & Control (245) & 21 & 100 & 124 & 75 & 119 & 51 & 1 & 207 & 37 & 1 & 31 & 213 & \\
\hline \multirow{2}{*}{ Aribam, 2020 [30] } & Case (60) & - & - & - & - & - & - & 22 & 25 & 13 & - & - & - & \multirow{2}{*}{0.158} \\
\hline & Control (60) & - & - & - & - & - & - & 26 & 23 & 11 & - & - & - & \\
\hline \multirow[t]{2}{*}{$\begin{array}{c}\text { First Author, } \\
\text { Publication Year }\end{array}$} & \multirow[t]{2}{*}{ Groups $(N)$} & \multicolumn{6}{|c|}{ FokI (rs2228570) } & \multicolumn{6}{|c|}{ BglI (rs739837) } & \multirow[t]{2}{*}{$p$-Value of HWE } \\
\hline & & \multicolumn{2}{|c|}{$\mathrm{FF}$} & \multicolumn{2}{|c|}{ Ff } & \multicolumn{2}{|c|}{$\mathrm{Ff}$} & \multicolumn{2}{|c|}{$\mathrm{BB}$} & \multicolumn{2}{|c|}{$\mathrm{Bb}$} & \multicolumn{2}{|c|}{$\mathrm{bb}$} & \\
\hline \multirow{2}{*}{ Barbosa, 2020 [31] } & Case (164 and 163) & \multicolumn{2}{|c|}{19} & 64 & & 8 & & 2 & & & & & & \\
\hline & Control (179 and 188) & 17 & & 80 & & 8 & & 4 & & & & & & 0.691 and 0.347 \\
\hline & Case (204 and 213) & 22 & & 85 & & 9 & & 6 & & & & & & \\
\hline Fattur1, 2020 [33] & Control (132 and 121) & 13 & & 63 & & 5 & & 3 & & & & & & 0.435 and 0.692 \\
\hline & Case (138 and 99) & 19 & & 60 & & 5 & & 1 & & & & & & \\
\hline Madalena, 2020 [35] & Control (19 and 12) & 2 & & 7 & & 1 & & 1 & & & & & & 0.649 and 0.665 \\
\hline
\end{tabular}

Abbreviation: HWE, Hardy-Weinberg equilibrium. AA, FF, TT, BB-homozygous dominant; Aa, Ff, Tt, Bb-heterozygous; aa, ff, bb-homozygous recessive.

\subsection{Meta-Analysis}

Table 5 shows the pooled analysis of the association between the ApaI (rs7975232) polymorphism and the risk of dental caries. The pooled ORs for allele, homozygote, heterozygote, recessive, and dominant were 0.89 (95\%CI: 0.70, 1.13; $p=0.34 ; \mathrm{I}^{2}=52 \%$, 0.86 (95\%CI: 0.49, 1.50; $\left.p=0.59 ; \mathrm{I}^{2}=57 \%\right], 0.83$ (95\%CI: 0.42, 1.62; $\left.p=0.58 ; \mathrm{I}^{2}=69 \%\right]$, 0.91 (95\%CI: 0.55, 1.50; $\left.p=0.71 ; \mathrm{I}^{2}=50 \%\right]$, and 0.87 (95\%CI: 0.69, $1.10 ; p=0.24 ; \mathrm{I}^{2}=8 \%$ ], re- 
spectively. These results indicate that there was no association between the ApaI (rs7975232) polymorphism and susceptibility to dental caries.

Table 5. The results of pooled analysis for association between ApaI (rs7975232) polymorphism and dental caries risk based on five genetic models.

\begin{tabular}{|c|c|c|c|c|c|c|c|}
\hline \multirow{2}{*}{ Genetic Model } & \multirow{2}{*}{$\begin{array}{c}\text { First Author, } \\
\text { Publication Year }\end{array}$} & \multicolumn{2}{|c|}{ Case } & \multicolumn{2}{|c|}{ Control } & \multirow{2}{*}{ Weight } & \multirow{2}{*}{$\begin{array}{c}\text { Odds Ratio } \\
\text { M-H, Random, } 95 \% \mathrm{CI}\end{array}$} \\
\hline & & Events & Total & Events & Total & & \\
\hline \multirow{3}{*}{ a vs. A } & Kong, 2017 [21] & 323 & 498 & 183 & 262 & $30.3 \%$ & $0.80[0.58,1.10]$ \\
\hline & $\mathrm{Yu}, 2017$ [37] & 249 & 400 & 273 & 400 & $33.4 \%$ & $0.77[0.57,1.03]$ \\
\hline & Qin, 2019 [36] & 445 & 608 & 348 & 490 & $36.4 \%$ & $1.11[0.85,1.45]$ \\
\hline Subtotal $(95 \% \mathrm{CI})$ & & & 1506 & & 1152 & $100.0 \%$ & $0.89[0.70,1.13]$ \\
\hline Total events & & 1017 & & 804 & & & \\
\hline \multicolumn{8}{|c|}{ Heterogeneity: $\mathrm{Tau}^{2}=0.02 ; \mathrm{Chi}^{2}=4.19, \mathrm{df}=2(P=0.12) ; \mathrm{I}^{2}=52 \% ;$ Test for overall effect: $Z=0.95(p=0.34)$} \\
\hline \multirow{3}{*}{ aa vs. AA } & Kong, 2017 [21] & 118 & 162 & 70 & 88 & $33.9 \%$ & $0.69[0.37,1.29]$ \\
\hline & $\mathrm{Yu}, 2017$ [37] & 82 & 115 & 97 & 121 & $34.8 \%$ & $0.61[0.34,1.12]$ \\
\hline & Qin, 2019 [36] & 158 & 175 & 124 & 145 & $31.3 \%$ & $1.57[0.80,3.11]$ \\
\hline Subtotal $(95 \% \mathrm{CI})$ & & & 452 & & 354 & $100.0 \%$ & $0.86[0.49,1.50]$ \\
\hline Total events & & 358 & & 291 & & & \\
\hline \multicolumn{8}{|c|}{ Heterogeneity: $\mathrm{Tau}^{2}=0.14 ; \mathrm{Chi}^{2}=4.68, \mathrm{df}=2(P=0.10) ; \mathrm{I}^{2}=57 \% ;$ Test for overall effect: $Z=0.54(p=0.59)$} \\
\hline \multirow{3}{*}{ Aa vs. AA } & Kong, 2017 [21] & 87 & 164 & 43 & 61 & $33.7 \%$ & $0.47[0.25,0.89]$ \\
\hline & $\mathrm{Yu}, 2017$ [37] & 85 & 118 & 79 & 103 & $34.4 \%$ & $0.78[0.43,1.44]$ \\
\hline & Qin, 2019 [36] & 129 & 146 & 100 & 121 & $31.8 \%$ & $1.59[0.80,3.18]$ \\
\hline Subtotal $(95 \%$ CI) & & & 428 & & 285 & $100.0 \%$ & $0.83[0.42,1.62]$ \\
\hline Total events & & 301 & & 222 & & & \\
\hline \multicolumn{8}{|c|}{ Heterogeneity: $\mathrm{Tau}^{2}=0.24 ; \mathrm{Chi}^{2}=6.50, \mathrm{df}=2(P=0.04) ; \mathrm{I}^{2}=69 \%$; Test for overall effect: $\mathrm{Z}=0.55(p=0.58)$} \\
\hline & Kong, $2017[21]$ & 205 & 249 & 113 & 131 & $34.0 \%$ & $0.74[0.41,1.34]$ \\
\hline \multirow{2}{*}{ aa + Aa vs. AA } & $\mathrm{Yu}, 2017[37]$ & 167 & 200 & 176 & 200 & $35.6 \%$ & $0.69[0.39,1.22]$ \\
\hline & Qin, 2019 [36] & 287 & 304 & 224 & 245 & $30.4 \%$ & $1.58[0.82,3.07]$ \\
\hline Subtotal $(95 \% \mathrm{CI})$ & & & 753 & & 576 & $100.0 \%$ & $0.91[0.55,1.50]$ \\
\hline Total events & & 659 & & 513 & & & \\
\hline \multicolumn{8}{|c|}{ Heterogeneity: $\mathrm{Tau}^{2}=0.10 ; \mathrm{Chi}^{2}=4.03, \mathrm{df}=2(P=0.13) ; \mathrm{I}^{2}=50 \%$; Test for overall effect: $\mathrm{Z}=0.37(p=0.71)$} \\
\hline \multirow{3}{*}{ aa vs. $\mathrm{AA}+\mathrm{Aa}$} & Kong, 2017 [21] & 118 & 249 & 70 & 131 & $28.2 \%$ & $0.78[0.51,1.20]$ \\
\hline & $\mathrm{Yu}, 2017$ [37] & 82 & 200 & 97 & 200 & $33.4 \%$ & $0.74[0.50,1.10]$ \\
\hline & Qin, 2019 [36] & 158 & 304 & 124 & 245 & $38.5 \%$ & $1.06[0.75,1.48]$ \\
\hline Subtotal $(95 \% \mathrm{CI})$ & & & 753 & & 576 & $100.0 \%$ & $0.87[0.69,1.10]$ \\
\hline Total events & & 358 & & 291 & & & \\
\hline \multicolumn{8}{|c|}{ Heterogeneity: $\mathrm{Chi}^{2}=2.16, \mathrm{df}=2(P=0.34) ; \mathrm{I}^{2}=8 \% ;$ Test for overall effect: $\mathrm{Z}=1.18(p=0.24)$} \\
\hline
\end{tabular}

Abbreviation: $\mathrm{CI}$, confidence interval.

Table 6 demonstrates that the f allele (0.58 (95\%CI: 0.38, 0.88); $p=0.01 ; \mathrm{I}^{2}=85 \%$ ), homozygote ( 0.52 (95\%CI: $\left.0.29,0.92 ; p=0.02 ; \mathrm{I}^{2}=66 \%\right)$, and dominant models $(0.53$ ( $95 \% \mathrm{CI}$ : $0.33,0.87 ; p=0.01 ; \mathrm{I}^{2}=64 \%$ ) of the FokI ( $\left.r s 10735810\right)$ ff genotype polymorphism had a protective role for the risk of dental caries, and the likelihood of caries in the individuals with these polymorphisms was approximately half that of those without these polymorphisms. The pooled ORs for other genetic models of FokI (rs10735810) polymorphisms (heterozygote and recessive) were not significant and the effect estimate was nearer to 1.

There was no association between the TaqI ( $r$ s731236) polymorphism and susceptibility to dental caries based on the five genetic models (Table 7).

Table 8 shows that the pooled ORs for allele, homozygote, heterozygote, recessive, and dominant were 0.92 (95\%CI: $0.58,1.46 ; p=0.73 ; \mathrm{I}^{2}=68 \%$ ], 3.89 (95\%CI: $0.16,95.85 ; p=0.41$ ], 2.71 (95\%CI: 0.11, 69.34; $p=0.55$ ], 3.74 (95\%CI: 0.15, 92.12; $p=0.42$ ], and 0.86 (95\%CI: $\left.0.48,1.54 ; p=0.61 ; \mathrm{I}^{2}=76 \%\right]$, respectively. Although the effect estimates for homozygote, heterozygote, and recessive models were $>1$, these estimates were derived from only one study each, and wider confidence intervals indicate that the sample sizes in these studies were very small. These findings indicate that there was no association between the BsmI (rs1544410) polymorphism and susceptibility to dental caries. 
Table 6. Meta-analysis for association between FokI (rs10735810) polymorphism and dental caries risk based on five genetic models.

\begin{tabular}{|c|c|c|c|c|c|c|c|}
\hline \multirow{2}{*}{ Genetic Model } & \multirow{2}{*}{$\begin{array}{c}\text { First Author, } \\
\text { Publication Year }\end{array}$} & \multicolumn{2}{|c|}{ Case } & \multicolumn{2}{|c|}{ Control } & \multirow{2}{*}{ Weight } & \multirow{2}{*}{$\begin{array}{c}\text { Odds Ratio } \\
\text { M-H, Random, } 95 \% \mathrm{Cl}\end{array}$} \\
\hline & & Events & Total & Events & Total & & \\
\hline \multirow{3}{*}{ fvs. F } & Kong, 2017 [21] & 228 & 498 & 131 & 262 & $32.7 \%$ & $0.84[0.63,1.14]$ \\
\hline & $\mathrm{Yu}, 2017$ [37] & 132 & 400 & 184 & 400 & $33.1 \%$ & $0.58[0.43,0.77]$ \\
\hline & Qin, 2019 [36] & 152 & 608 & 221 & 490 & $34.2 \%$ & $0.41[0.31,0.52]$ \\
\hline Subtotal (95\%CI) & & & 1506 & & 1152 & $100.0 \%$ & $0.58[0.38,0.88]$ \\
\hline Total events & & 512 & & 536 & & & \\
\hline \multicolumn{8}{|c|}{ Heterogeneity: $\mathrm{Tau}^{2}=0.12 ; \mathrm{Chi}^{2}=13.37, \mathrm{df}=2(P=0.001) ; \mathrm{I}^{2}=85 \% ;$ Test for overall effect: $Z=2.56(p=0.01)$} \\
\hline & Kong, 2017 [21] & 48 & 117 & 34 & 68 & $32.3 \%$ & $0.70[0.38,1.27]$ \\
\hline \multirow[t]{2}{*}{ ff vs. FF } & $\mathrm{Yu}, 2017$ [37] & 18 & 104 & 49 & 114 & $31.3 \%$ & $0.28[0.15,0.52]$ \\
\hline & Qin, 2019 [36] & 46 & 144 & 51 & 126 & $36.4 \%$ & $0.69[0.42,1.14]$ \\
\hline Subtotal (95\%CI) & & & 365 & & 308 & $100.0 \%$ & $0.52[0.29,0.92]$ \\
\hline Total events & & 112 & & 134 & & & \\
\hline \multicolumn{8}{|c|}{ Heterogeneity: $\mathrm{Tau}^{2}=0.17 ; \mathrm{Chi}^{2}=5.91, \mathrm{df}=2(P=0.05) ; \mathrm{I}^{2}=66 \% ;$ Test for overall effect: $\mathrm{Z}=2.24(p=0.02)$} \\
\hline & Kong, 2017 [21] & 132 & 201 & 63 & 97 & $23.3 \%$ & $1.03[0.62,1.72]$ \\
\hline \multirow[t]{2}{*}{ Ff vs. FF } & $\mathrm{Yu}, 2017$ [37] & 96 & 182 & 86 & 151 & $35.5 \%$ & $0.84[0.55,1.30]$ \\
\hline & Qin, 2019 [36] & 160 & 258 & 119 & 194 & $41.2 \%$ & $1.03[0.70,1.51]$ \\
\hline Subtotal $(95 \% \mathrm{CI})$ & & & 641 & & 442 & $100.0 \%$ & $0.96[0.75,1.24]$ \\
\hline Total events & & 388 & & 268 & & & \\
\hline \multicolumn{8}{|c|}{ Heterogeneity: $\mathrm{Chi}^{2}=0.54, \mathrm{df}=2(P=0.76) ; \mathrm{I}^{2}=0 ;$ Test for overall effect: $Z=0.29(p=0.77)$} \\
\hline \multirow{3}{*}{$\mathrm{ff}+$ Aa vs. FF } & Kong, 2017 [21] & 180 & 249 & 97 & 131 & $22.9 \%$ & $0.91[0.57,1.48]$ \\
\hline & $\mathrm{Yu}, 2017[37]$ & 114 & 200 & 135 & 200 & $37.7 \%$ & $0.64[0.42,0.96]$ \\
\hline & Qin, 2019 [36] & 206 & 304 & 170 & 245 & $39.4 \%$ & $0.93[0.65,1.33]$ \\
\hline Subtotal $(95 \% \mathrm{CI})$ & & & 753 & & 576 & $100.0 \%$ & $0.82[0.64,1.04]$ \\
\hline Total events & & 500 & & 402 & & & \\
\hline \multicolumn{8}{|c|}{ Heterogeneity: $\mathrm{Chi}^{2}=2.09, \mathrm{df}=2(P=0.35) ; \mathrm{I}^{2}=4 \% ;$ Test for overall effect: $\mathrm{Z}=1.66(p=0.10)$} \\
\hline \multirow{3}{*}{ ff vs. FF + Ff } & Kong, 2017 [21] & 48 & 249 & 34 & 131 & $33.6 \%$ & $0.68[0.41,1.13]$ \\
\hline & $\mathrm{Yu}, 2017$ [37] & 18 & 200 & 49 & 200 & $29.9 \%$ & $0.30[0.17,0.55]$ \\
\hline & Qin, 2019 [36] & 46 & 304 & 51 & 245 & $36.6 \%$ & $0.68[0.44,1.05]$ \\
\hline Subtotal (95\%CI) & & & 753 & & 576 & $100.0 \%$ & $0.53[0.33,0.87]$ \\
\hline Total events & & & 753 & & 576 & $100.0 \%$ & $0.53[0.33,0.87]$ \\
\hline \multicolumn{8}{|c|}{ Heterogeneity: $\mathrm{Tau}^{2}=0.12 ; \mathrm{Chi}^{2}=5.53, \mathrm{df}=2(P=0.06) ; \mathrm{I}^{2}=64 \%$; Test for overall effect: $Z=2.53(p=0.01)$} \\
\hline
\end{tabular}

Abbreviation: $\mathrm{CI}$, confidence interval.

Table 7. Association between TaqI (rs731236) polymorphism and dental caries risk based on five genetic models.

\begin{tabular}{|c|c|c|c|c|c|c|c|}
\hline \multirow{2}{*}{ Genetic Model } & \multirow{2}{*}{$\begin{array}{c}\text { First Author, } \\
\text { Publication Year }\end{array}$} & \multicolumn{2}{|c|}{ Case } & \multicolumn{2}{|c|}{ Control } & \multirow{2}{*}{ Weight } & \multirow{2}{*}{$\begin{array}{c}\text { Odds Ratio } \\
\text { M-H, Random, } 95 \% \mathrm{CI}\end{array}$} \\
\hline & & Events & Total & Events & Total & & \\
\hline \multirow{6}{*}{ t vs. T } & Cogulu, 2016 [32] & 108 & 224 & 32 & 76 & $7.3 \%$ & $1.28[0.76,2.16]$ \\
\hline & Holla, 2017 [34] & 170 & 470 & 119 & 306 & $27.3 \%$ & $0.89[0.66,1.20]$ \\
\hline & Kong, 2017 [21] & 19 & 498 & 11 & 262 & $4.1 \%$ & $0.91[0.42,1.93]$ \\
\hline & Yu, 2017 [37] & 29 & 400 & 42 & 400 & $11.6 \%$ & $0.67[0.41,1.09]$ \\
\hline & Qin, 2019 [36] & 332 & 608 & 281 & 490 & $42.0 \%$ & $0.89[0.70,1.14]$ \\
\hline & Aribam, 2020 [30] & 51 & 120 & 45 & 120 & $7.7 \%$ & $1.23[0.73,2.07]$ \\
\hline Subtotal (95\%CI) & & & 2320 & & 1654 & $100.0 \%$ & $0.92[0.79,1.08]$ \\
\hline Total events & & 709 & & 530 & & & \\
\hline \multicolumn{8}{|c|}{ Heterogeneity: $\mathrm{Chi}^{2}=4.47, \mathrm{df}=5(P=0.48) ; \mathrm{I}^{2}=0 \% ;$ Test for overall effect: $\mathrm{Z}=1.03(p=0.30)$} \\
\hline & Cogulu, 2016 [32] & 31 & 66 & 9 & 24 & $22.2 \%$ & $1.48[0.57,3.85]$ \\
\hline \multirow{4}{*}{ tt vs. TT } & Holla, 2017 [34] & 30 & 125 & 17 & 68 & $53.1 \%$ & $0.95[0.48,1.88]$ \\
\hline & Kong, 2017 [21] & 0 & 230 & 0 & 120 & & Not estimable \\
\hline & $\mathrm{Yu}, 2017$ [37] & 0 & 171 & 0 & 158 & & Not estimable \\
\hline & Qin, 2019 [36] & 29 & 30 & 37 & 38 & $3.4 \%$ & $0.78[0.05,13.07]$ \\
\hline & Aribam, 2020 [30] & 13 & 35 & 11 & 37 & $21.3 \%$ & $1.40[0.52,3.73]$ \\
\hline Subtotal (95\%CI) & & & 657 & & 445 & $100.0 \%$ & $1.15[0.72,1.86]$ \\
\hline Total events & & 103 & & 74 & & & \\
\hline \multicolumn{8}{|c|}{ Heterogeneity: $\mathrm{Chi}^{2}=0.79, \mathrm{df}=3(P=0.85) ; \mathrm{I}^{2}=0 \%$; Test for overall effect: $\mathrm{Z}=0.58(p=0.56)$} \\
\hline
\end{tabular}


Table 7. Cont.

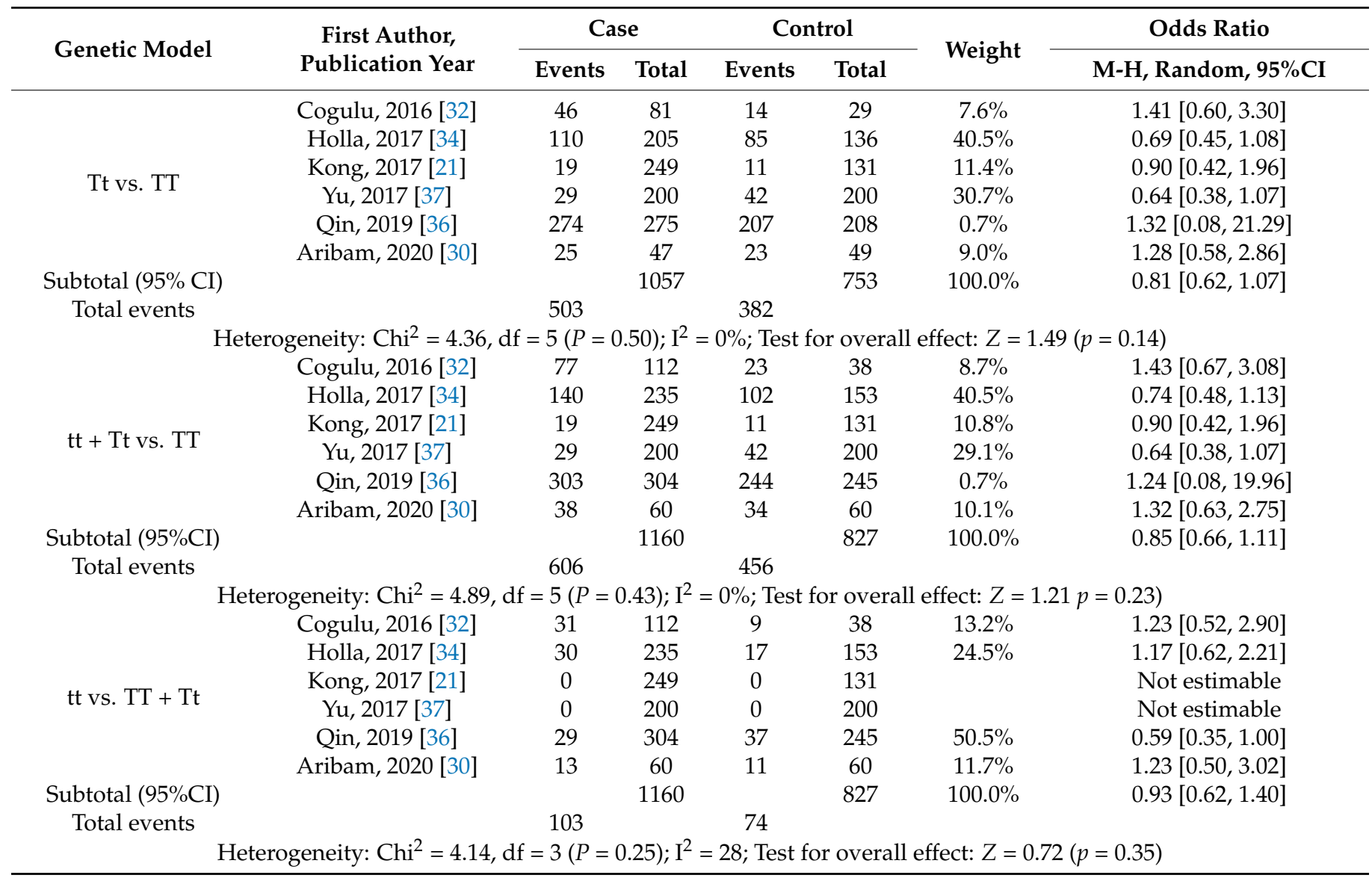

Abbreviation: $\mathrm{CI}$, confidence interval.

Table 8. The results of meta-analysis exploring the association between BsmI (rs1544410) polymorphism and dental caries risk based on five genetic models.

\begin{tabular}{|c|c|c|c|c|c|c|c|}
\hline \multirow{2}{*}{ Genetic Model } & \multirow{2}{*}{$\begin{array}{c}\text { First Author, } \\
\text { Publication Year }\end{array}$} & \multicolumn{2}{|c|}{ Case } & \multicolumn{2}{|c|}{ Control } & \multirow{2}{*}{ Weight } & \multirow{2}{*}{$\begin{array}{c}\text { Odds ratio } \\
\mathrm{M}-\mathrm{H}, \text { Random, } 95 \% \mathrm{CI}\end{array}$} \\
\hline & & Events & Total & Events & Total & & \\
\hline \multirow{3}{*}{ b vs. B } & Kong, 2017 [21] & 346 & 498 & 202 & 262 & $38.6 \%$ & $0.68[0.48,0.96]$ \\
\hline & $\mathrm{Yu}, 2017$ [37] & 364 & 400 & 369 & 400 & $31.1 \%$ & $0.85[0.51,1.40]$ \\
\hline & Qin, 2019 [36] & 580 & 608 & 457 & 490 & $30.3 \%$ & $1.50[0.89,2.51]$ \\
\hline Subtotal $(95 \% \mathrm{CI})$ & & & 1506 & & 1152 & $100.0 \%$ & $0.92[0.58,1.46]$ \\
\hline Total events & & 1290 & & 1028 & & & \\
\hline \multicolumn{8}{|c|}{ Heterogeneity: $\mathrm{Tau}^{2}=0.11 ; \mathrm{Chi}^{2}=6.24, \mathrm{df}=2(P=0.04) ; \mathrm{I}^{2}=68 \%$; Test for overall effect: $\mathrm{Z}=0.34(p=0.73)$} \\
\hline & Kong, 2017 [21] & 97 & 97 & 71 & 71 & & Not estimable \\
\hline \multirow[t]{2}{*}{ bb vs. BB } & $\mathrm{Yu}, 2017[37]$ & 164 & 164 & 169 & 169 & & Not estimable \\
\hline & Qin, 2019 [36] & 276 & 276 & 213 & 214 & $100.0 \%$ & $3.89[0.16,95.85]$ \\
\hline Subtotal (95\%CI) & & & 537 & & 454 & $100.0 \%$ & $3.89[0.16,95.85]$ \\
\hline \multirow[t]{3}{*}{ Total events } & & 537 & & 453 & & & \\
\hline & Heterogeneity: & applicab & $\mathrm{e}$; Test & r overall & fect: $Z$ & $83(p=0.4$ & \\
\hline & Kong, 2017 [21] & 152 & 152 & 60 & 60 & & Not estimable \\
\hline \multirow[t]{2}{*}{ Bb vs. BB } & $\mathrm{Yu}, 2017$ [37] & 36 & 36 & 31 & 31 & & Not estimable \\
\hline & Qin, 2019 [36] & 28 & 28 & 31 & 32 & $100.0 \%$ & $2.71[0.11,69.34]$ \\
\hline \multirow{3}{*}{$\begin{array}{l}\text { Subtotal }(95 \% \mathrm{CI}) \\
\text { Total events }\end{array}$} & & & 216 & & 123 & $100.0 \%$ & $2.71[0.11,69.34]$ \\
\hline & & 216 & & 122 & & & \\
\hline & Heterogeneity: & applicab & $\mathrm{e}$; Test & r overall & fect: $Z$ & $60(p=0$. & \\
\hline
\end{tabular}


Table 8. Cont.

\begin{tabular}{|c|c|c|c|c|c|c|c|}
\hline \multirow{2}{*}{ Genetic Model } & \multirow{2}{*}{$\begin{array}{c}\text { First Author, } \\
\text { Publication Year }\end{array}$} & \multicolumn{2}{|c|}{ Case } & \multicolumn{2}{|c|}{ Control } & \multirow{2}{*}{ Weight } & \multirow{2}{*}{$\begin{array}{c}\text { Odds ratio } \\
\text { M-H, Random, } 95 \% \mathrm{CI}\end{array}$} \\
\hline & & Events & Total & Events & Total & & \\
\hline \multirow{3}{*}{$b b+B b$ vs. $B B$} & Kong, 2017 [21] & 249 & 249 & 131 & 131 & & Not estimable \\
\hline & $\mathrm{Yu}, 2017$ [37] & 200 & 200 & 200 & 200 & & Not estimable \\
\hline & Qin, 2019 [36] & 304 & 304 & 244 & 245 & $100.0 \%$ & $3.74[0.15,92.12]$ \\
\hline Subtotal (95\%CI) & & & 753 & & 576 & $100.0 \%$ & $3.74[0.15,92.12]$ \\
\hline \multirow[t]{3}{*}{ Total events } & & 753 & & 575 & & & \\
\hline & Heterogeneity: & applicab & e; Test $f$ & r overall & G 7 & 01 (n 01 & \\
\hline & Kong, 2017 [21] & 97 & 249 & 71 & 131 & $35.6 \%$ & $0.54[0.35,0.83]$ \\
\hline \multirow[t]{2}{*}{ bb vs. $\mathrm{BB}+\mathrm{Bb}$} & $\mathrm{Yu}, 2017$ [37] & 164 & 200 & 169 & 200 & $32.4 \%$ & $0.84[0.49,1.41]$ \\
\hline & Qin, 2019 [36] & 276 & 304 & 213 & 245 & $32.0 \%$ & $1.48[0.86,2.54]$ \\
\hline \multicolumn{2}{|l|}{ Subtotal $(95 \% \mathrm{CI})$} & & 753 & & 576 & $100.0 \%$ & $0.86[0.48,1.54]$ \\
\hline \multicolumn{2}{|c|}{ Total events } & 537 & & 453 & & & \\
\hline \multicolumn{8}{|c|}{ Heterogeneity: $\mathrm{Tau}^{2}=0.20 ; \mathrm{Chi}^{2}=8.32, \mathrm{df}=2(P=0.02) ; \mathrm{I}^{2}=76 \%$; Test for overall effect: $Z=0.51(p=0.61)$} \\
\hline
\end{tabular}

Abbreviation: $\mathrm{CI}$, confidence interval.

Table 9 demonstrates that there was no association between the FokI (rs2228570) polymorphism and susceptibility to dental caries and there was a lack of heterogeneity between the studies $\left(\mathrm{I}^{2}=0 \%\right)$ in all five genetic models. The odds ratio for most of these models was closer to 1 , with narrow confidence intervals indicating no association.

Table 9. Results exploring the association between FokI (rs2228570) polymorphism and dental caries risk based on five genetic models.

\begin{tabular}{|c|c|c|c|c|c|c|c|}
\hline \multirow{2}{*}{ Genetic Model } & \multirow{2}{*}{$\begin{array}{c}\text { First Author, } \\
\text { Publication Year }\end{array}$} & \multicolumn{2}{|c|}{ Case } & \multicolumn{2}{|c|}{ Control } & \multirow{2}{*}{ Weight } & \multirow{2}{*}{$\frac{\text { Odds Ratio }}{\text { M-H, Random, } 95 \% \mathrm{CI}}$} \\
\hline & & Events & Total & Events & Total & & \\
\hline \multirow{3}{*}{ f vs. F } & Barbosa, 2020 [31] & 226 & 328 & 244 & 358 & $46.3 \%$ & $1.04[0.75,1.43]$ \\
\hline & Fatturi, 2020 [33] & 279 & 408 & 175 & 264 & $42.9 \%$ & $1.10[0.79,1.53]$ \\
\hline & Madalena, 2020 [35] & 178 & 276 & 27 & 38 & $10.8 \%$ & $0.74[0.35,1.56]$ \\
\hline Subtotal (95\%CI) & & & 1012 & & 660 & $100.0 \%$ & $1.03[0.83,1.28]$ \\
\hline Total events & & 683 & & 446 & & & \\
\hline \multicolumn{8}{|c|}{ Heterogeneity: $\mathrm{Chi}^{2}=0.91, \mathrm{df}=2(P=0.63) ; \mathrm{I}^{2}=0 \%$; Test for overall effect: $Z=0.29(p=0.77)$} \\
\hline & Barbosa, 2020 [31] & 81 & 100 & 82 & 99 & $47.5 \%$ & $0.88[0.43,1.82]$ \\
\hline \multirow[t]{2}{*}{ ff vs. FF } & Fatturi, 2020 [33] & 97 & 119 & 56 & 69 & $39.7 \%$ & $1.02[0.48,2.19]$ \\
\hline & Madalena, 2020 [35] & 59 & 78 & 10 & 12 & $12.8 \%$ & $0.62[0.12,3.09]$ \\
\hline Subtotal $(95 \% \mathrm{CI})$ & & & 297 & & 180 & $100.0 \%$ & $0.91[0.55,1.50]$ \\
\hline Total events & & 237 & & 148 & & & \\
\hline \multicolumn{8}{|c|}{ Heterogeneity: $\mathrm{Chi}^{2}=0.32, \mathrm{df}=2(P=0.85) ; \mathrm{I}^{2}=0 \% ;$ Test for overall effect: $Z=0.37(p=0.71)$} \\
\hline \multirow{3}{*}{ Ff vs. FF } & Barbosa, 2020 [31] & 64 & 83 & 80 & 97 & $48.2 \%$ & $0.72[0.34,1.49]$ \\
\hline & Fatturi, 2020 [33] & 85 & 107 & 63 & 76 & $43.2 \%$ & $0.80[0.37,1.70]$ \\
\hline & Madalena, 2020 [35] & 60 & 79 & 7 & 9 & $8.6 \%$ & $0.90[0.17,4.72]$ \\
\hline Subtotal $(95 \%$ CI) & & & 269 & & 182 & $100.0 \%$ & $0.77[0.46,1.27]$ \\
\hline Total events & & 209 & & 150 & & & \\
\hline \multicolumn{8}{|c|}{ Heterogeneity: $\mathrm{Chi}^{2}=0.08, \mathrm{df}=2(P=0.96) ; \mathrm{I}^{2}=0 \%$; Test for overall effect: $Z=1.04(p=0.30)$} \\
\hline \multirow{3}{*}{$\mathrm{ff}+\mathrm{Ff}$ vs. FF } & Barbosa, 2020 [31] & 145 & 164 & 162 & 179 & $47.7 \%$ & $0.80[0.40,1.60]$ \\
\hline & Fatturi, $2020[33]$ & 182 & 204 & 119 & 132 & $41.4 \%$ & $0.90[0.44,1.86]$ \\
\hline & Madalena, 2020 [35] & 119 & 138 & 17 & 19 & $10.9 \%$ & $0.74[0.16,3.45]$ \\
\hline Subtotal $(95 \% \mathrm{CI})$ & & & 506 & & 330 & $100.0 \%$ & $0.84[0.52,1.35]$ \\
\hline Total events & & 446 & & 298 & & & \\
\hline \multicolumn{8}{|c|}{ Heterogeneity: $\mathrm{Chi}^{2}=0.09, \mathrm{df}=2(P=0.96) ; \mathrm{I}^{2}=0 \%$; Test for overall effect: $Z=0.73(p=0.46)$} \\
\hline \multirow{3}{*}{ ff vs. FF + Ff } & Barbosa, 2020 [31] & 81 & 164 & 82 & 179 & $46.5 \%$ & $1.15[0.76,1.76]$ \\
\hline & Fatturi, 2020 [33] & 97 & 204 & 56 & 132 & $41.8 \%$ & $1.23[0.79,1.91]$ \\
\hline & Madalena, 2020 [35] & 59 & 138 & 10 & 19 & $11.8 \%$ & $0.67[0.26,1.76]$ \\
\hline Subtotal (95\%CI) & & & 506 & & 330 & $100.0 \%$ & $1.13[0.84,1.51]$ \\
\hline Total events & & 237 & & 148 & & & \\
\hline \multicolumn{8}{|c|}{ Heterogeneity: $\mathrm{Chi}^{2}=1.27, \mathrm{df}=2(P=0.53) ; \mathrm{I}^{2}=0 \%$; Test for overall effect: $\mathrm{Z}=0.82(p=0.41)$} \\
\hline
\end{tabular}


The pooled ORs for allele, homozygote, heterozygote, recessive, and dominant were 1.06 (95\%CI: 0.86, 1.31; $p=0.61 ; \mathrm{I}^{2}=0 \%$ ], 1.15 (95\%CI: 0.75, $\left.1.75 ; p=0.53 ; \mathrm{I}^{2}=0 \%\right]$, 1.15 (95\%CI: 0.79, 1.67; $\left.p=0.48 ; \mathrm{I}^{2}=0 \%\right], 1.14$ (95\%CI: $\left.0.80,1.62 ; p=0.46 ; \mathrm{I}^{2}=0 \%\right]$, and 1.02 (95\%CI: $0.73,1.42 ; p=0.91 ; \mathrm{I}^{2}=0 \%$, respectively (Table 10). There was no association between the $B g l I$ ( $r s 739837$ ) polymorphism and susceptibility to dental caries.

Table 10. The results from meta-analysis of the association between BglI (rs739837) polymorphism and dental caries risk based on five genetic models.

\begin{tabular}{|c|c|c|c|c|c|c|c|}
\hline \multirow{2}{*}{ Genetic Model } & \multirow{2}{*}{$\begin{array}{c}\text { First Author, } \\
\text { Publication Year }\end{array}$} & \multicolumn{2}{|c|}{ Case } & \multicolumn{2}{|c|}{ Control } & \multirow{2}{*}{ Weight } & \multirow{2}{*}{$\begin{array}{c}\text { Odds Ratio } \\
\text { M-H, Random, } 95 \% \mathrm{CI}\end{array}$} \\
\hline & & Events & Total & Events & Total & & \\
\hline \multirow{3}{*}{ b vs. B } & Barbosa, 2020 [31] & 186 & 326 & 203 & 376 & $48.1 \%$ & $1.13[0.84,1.53]$ \\
\hline & Fatturi, 2020 [33] & 199 & 426 & 112 & 242 & $45.2 \%$ & $1.02[0.74,1.40]$ \\
\hline & Madalena, 2020 [35] & 120 & 198 & 16 & 24 & $6.7 \%$ & $0.77[0.31,1.88]$ \\
\hline Subtotal (95\%CI) & & & 950 & & 642 & $100.0 \%$ & $1.06[0.86,1.31]$ \\
\hline Total events & & 505 & & 331 & & & \\
\hline \multicolumn{8}{|c|}{ Heterogeneity: $\mathrm{Chi}^{2}=0.74, \mathrm{df}=2(P=0.69) ; \mathrm{I}^{2}=0 \%$; Test for overall effect: $Z=0.51(p=0.61)$} \\
\hline & Barbosa, 2020 [31] & 52 & 81 & 58 & 101 & $45.8 \%$ & $1.33[0.73,2.43]$ \\
\hline \multirow[t]{2}{*}{ bb vs. BB } & Fatturi, 2020 [33] & 49 & 112 & 27 & 63 & $48.1 \%$ & $1.04[0.56,1.93]$ \\
\hline & Madalena, 2020 [35] & 34 & 47 & 5 & 6 & $6.1 \%$ & $0.52[0.06,4.91]$ \\
\hline Subtotal $(95 \% \mathrm{CI})$ & & & 240 & & 170 & $100.0 \%$ & $1.15[0.75,1.75]$ \\
\hline Total events & & 135 & & & & & \\
\hline \multicolumn{8}{|c|}{ Heterogeneity: $\mathrm{Chi}^{2}=0.80, \mathrm{df}=2(P=0.67) ; \mathrm{I}^{2}=0 \% ;$ Test for overall effect: $\mathrm{Z}=0.63(p=0.53)$} \\
\hline \multirow{3}{*}{ Bb vs. BB } & Barbosa, 2020 [31] & 82 & 111 & 87 & 130 & $40.7 \%$ & $1.40[0.80,2.44]$ \\
\hline & Fatturi, 2020 [33] & 101 & 164 & 58 & 94 & $55.1 \%$ & $1.00[0.59,1.68]$ \\
\hline & Madalena, 2020 [35] & 52 & 65 & 6 & 7 & $4.2 \%$ & $0.67[0.07,6.03]$ \\
\hline Subtotal $(95 \% \mathrm{CI})$ & & & 340 & & 231 & $100.0 \%$ & $1.15[0.79,1.67]$ \\
\hline Total events & & 235 & & 151 & & & \\
\hline \multicolumn{8}{|c|}{ Heterogeneity: $\mathrm{Chi}^{2}=1.00, \mathrm{df}=2(P=0.61) ; \mathrm{I}^{2}=0 \%$; Test for overall effect: $Z=0.71(p=0.48)$} \\
\hline & Barbosa, 2020 [31] & 134 & 163 & 145 & 188 & $40.9 \%$ & $1.37[0.81,2.32]$ \\
\hline \multirow[t]{2}{*}{$\mathrm{bb}+\mathrm{Bb}$ vs. BB } & Fatturi, 2020 [33] & 150 & 213 & 85 & 121 & $54.7 \%$ & $1.01[0.62,1.64]$ \\
\hline & Madalena, $2020[35]$ & 86 & 99 & 11 & 12 & $4.4 \%$ & $0.60[0.07,5.05]$ \\
\hline Subtotal $(95 \% \mathrm{CI})$ & & & 475 & & 321 & $100.0 \%$ & $1.14[0.80,1.62]$ \\
\hline Total events & & & & & & & \\
\hline \multicolumn{8}{|c|}{ Heterogeneity: $\mathrm{Chi}^{2}=1.06, \mathrm{df}=2(P=0.59) ; \mathrm{I}^{2}=0 \% ;$ Test for overall effect: $Z=0.73(p=0.46)$} \\
\hline \multirow{3}{*}{ bb vs. $\mathrm{BB}+\mathrm{Bb}$} & Barbosa, 2020 [31] & 52 & 163 & 58 & 188 & $53.1 \%$ & $1.05[0.67,1.65]$ \\
\hline & Fatturi, $2020[33]$ & 49 & 213 & 27 & 121 & $38.4 \%$ & $1.04[0.61,1.77]$ \\
\hline & Madalena, 2020 [35] & 34 & 99 & 5 & 12 & $8.5 \%$ & $0.73[0.22,2.48]$ \\
\hline Subtotal (95\%CI) & & & 475 & & 321 & $100.0 \%$ & $1.02[0.73,1.42]$ \\
\hline Total events & & 135 & & & & & \\
\hline \multicolumn{8}{|c|}{ Heterogeneity: $\mathrm{Chi}^{2}=0.30, \mathrm{df}=2(P=0.86) ; \mathrm{I}^{2}=0 \%$; Test for overall effect: $\mathrm{Z}=0.11(p=0.91)$} \\
\hline
\end{tabular}

Abbreviation: CI, confidence interval.

\subsection{Subgroup Analysis}

As there was an adequate number of studies on the TaqI (rs731236) polymorphism, subgroup analyses in relation to ethnicity and genotyping were conducted (Table 11). The overall effect still remained insignificant with none of the subgroups demonstrating any association between the TaqI (rs731236) polymorphism and susceptibility to dental caries across the five genetic models.

Table 11. Subgroup analyses based on ethnicity and genotyping method for TaqI (rs731236) polymorphism.

\begin{tabular}{|c|c|c|c|c|c|}
\hline Variable (N) & $\begin{array}{c}\text { t vs. T } \\
\text { OR }(95 \% \mathrm{CI}), p, \mathrm{I}^{2}\end{array}$ & $\begin{array}{c}\text { tt vs. TT } \\
\text { OR }(95 \% \mathrm{CI}), p, \mathrm{I}^{2}\end{array}$ & $\begin{array}{c}\text { Tt vs. TT } \\
\text { OR }(95 \% \mathrm{CI}), p, \mathrm{I}^{2}\end{array}$ & $\begin{array}{c}\mathrm{tt}+\mathrm{Tt} \text { vs. TT } \\
\text { OR }(95 \% \mathrm{CI}), p, \mathrm{I}^{2}\end{array}$ & $\begin{array}{c}\mathrm{tt} \text { vs. TT + Tt } \\
\text { OR }(95 \% \mathrm{CI}), p, \mathrm{I}^{2}\end{array}$ \\
\hline \multicolumn{6}{|l|}{ Ethnicity } \\
\hline Caucasian (3) & $1.02(0.81,1.29), 0.86,2 \%$ & $1.17(0.72,1.89), 0.53,0 \%$ & $0.96(0.59,1.56), 0.87,36 \%$ & $1.02(0.64,1.61), 0.94,39 \%$ & $1.20(0.77,1.87), 0.42,0 \%$ \\
\hline Asian (3) & $0.85(0.69,1.05), 0.13,0 \%$ & $0.75(0.05,13.07), 0.87$ & $0.72(0.47,1.10), 0.13,0 \%$ & $0.72(0.47,1.10), 0.13,0 \%$ & $0.59(0.35,1.00), 0.05$ \\
\hline \multicolumn{6}{|l|}{ Genotyping method } \\
\hline PCR (4) & $0.99(0.71,1.37), 0.95,27 \%$ & $1.44(0.72,2.85), 0.30,0 \%$ & $0.91(0.62,1.33), 0.63,14 \%$ & $0.96(0.64,1.43), 0.83,28 \%$ & $1.23(0.66,2.29), 0.51,0 \%$ \\
\hline TaqMan (2) & $0.89(0.74,1.08), 0.23,0 \%$ & $0.94(0.48,1.82), 0.85,0 \%$ & $0.71(0.46,1.09), 0.12,0 \%$ & $0.75(0.49,1.14), 0.17,0 \%$ & $0.81(0.42,1.58), 0.54,62 \%$ \\
\hline
\end{tabular}




\subsection{Sensitivity Analysis}

We conducted "cumulative analysis" and "one study removed" analyses to evaluate the stability of the findings related to six polymorphisms. The results show that the results were consistent/stable for the six polymorphisms. Additionally, for the TaqI (rs731236) polymorphism, we removed two studies $[34,36]$ reporting an HWE deviation in the control group and found that the pooled ORs still remained the same.

\subsection{Publication Bias}

The funnel plots (Figure 2) and $p>0.05$ for both Egger's and Begg's tests demonstrate a lack of publication bias with regard to all six polymorphisms considered in this review.

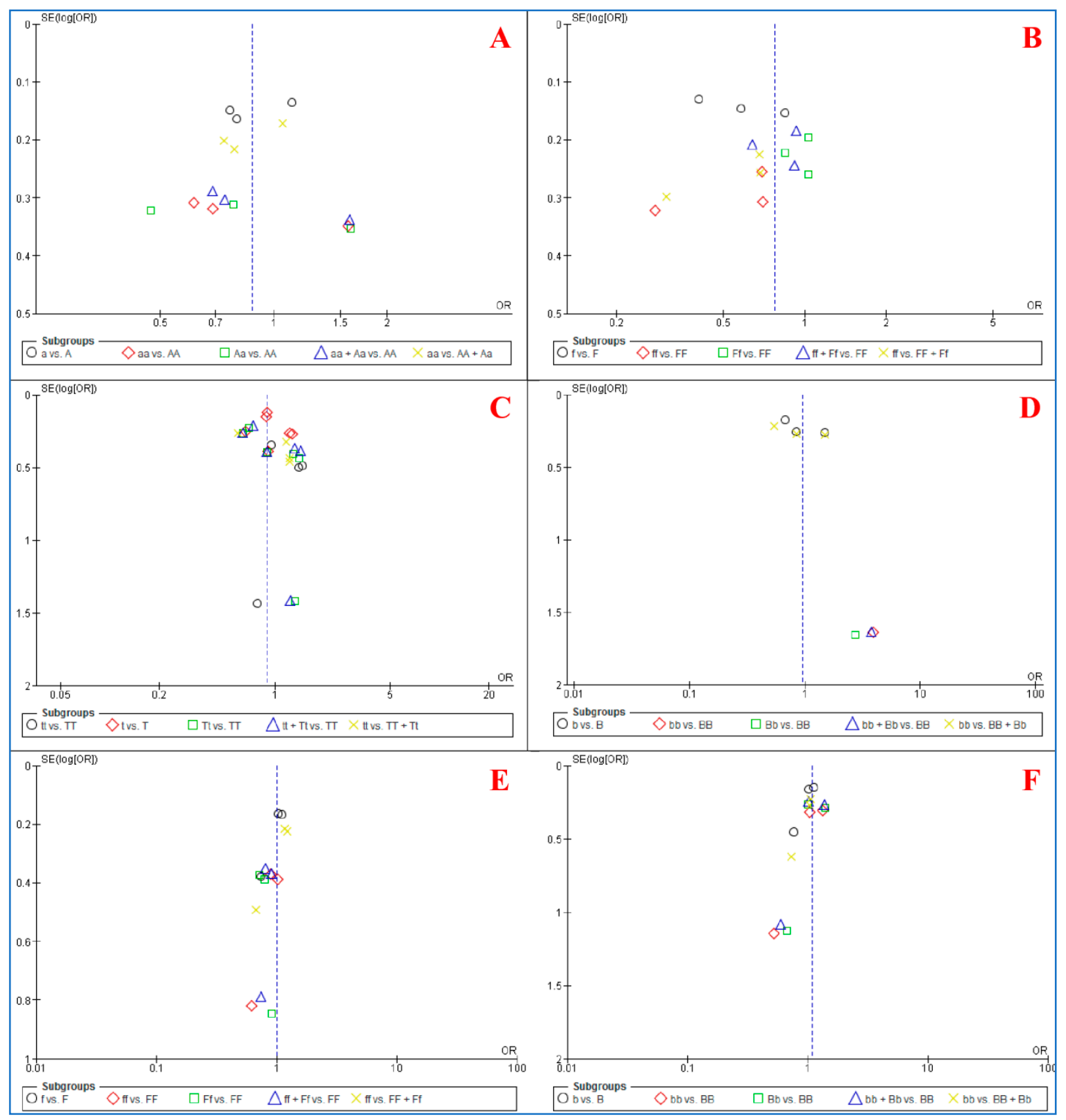

Figure 2. Funnel plots for association between six polymorphisms of vitamin D receptor $(V D R)$ and dental caries risk based on five genetic. (A): ApaI (rs7975232). (B): FokI (rs10735810). (C): TaqI (rs731236). (D): BsmI (rs1544410). (E): FokI (rs2228570). (F): BglI (rs739837) 


\section{Discussion}

The present meta-analysis evaluated the association between $V D R$ polymorphisms (ApaI (rs7975232), FokI (rs10735810), TaqI (rs731236), BsmI (rs1544410), FokI (rs2228570), and $\mathrm{BglI}$ (rs739837)) and the risk of dental caries in children. None of the polymorphisms were associated with the risk of dental caries, except for the FokI (rs10735810) polymorphism, with the $\mathrm{f}$ allele and $\mathrm{ff}$ genotype of this polymorphism having a protective role in dental caries occurrence.

The role of genetic factors in the risk of dental caries is still largely unknown despite numerous studies. Dental caries is a multifactorial disease caused by interactions between environmental factors, behavioral factors, several genetic factors, and gene-environment interactions [31]. Advances in transcriptional research have provided a variety of data on the interaction between $V D R$ and other transcriptionally active proteins, demonstrating the potential of $V D R$ to exert a wide range of biological reactions [38]. Vitamin D is known as a modulator of calcium homeostasis and plays an important role in regulating electrolytes and blood pressure. Evidence has shown that the most active metabolite of this vitamin can regulate the immune response and also has anti-inflammatory activity [39]. VDR gene polymorphisms have been shown to be strongly related to mineral density $[32,40,41]$ and a meta-analysis [42] confirmed this. Although results from individual studies remain inconsistent, a meta-analysis of controlled clinical trials showed that early vitamin D supplementation could reduce the risk of dental caries by $47-54 \%$ [20]. Although the mechanism of action is unknown, $V D R$ gene polymorphisms could modulate the effect of vitamin D supplementation. For instance, one study found some VDR polymorphisms to modify the association of vitamin D supplementation with the risk of a specific type of cancer [43]. The role of $V D R$ polymorphisms in modifying the effect of vitamin D supplementation on dental caries needs further exploration.

$V D R$ plays an important role in regulating the expression of genes associated with the immune response, calcium homeostasis, and cell differentiation and proliferation [18]. The distribution of $V D R$ polymorphisms could show different patterns based on ethnicities and age [44-47]. Research has shown ethnic differences in vitamin D status and their correlation to hormonal homeostasis and bone phenotype, as well as the influence of environmental factors such as lifestyle, diet, and sun exposure [17,18]. However, we could not find any differences based on ethnicities in this meta-analysis.

Our meta-analysis showed a protective role of the FokI (rs10735810) polymorphism on dental caries. This might be due to its interactions with co-transcription factors [18] and its location (Figure 3) [18,48].

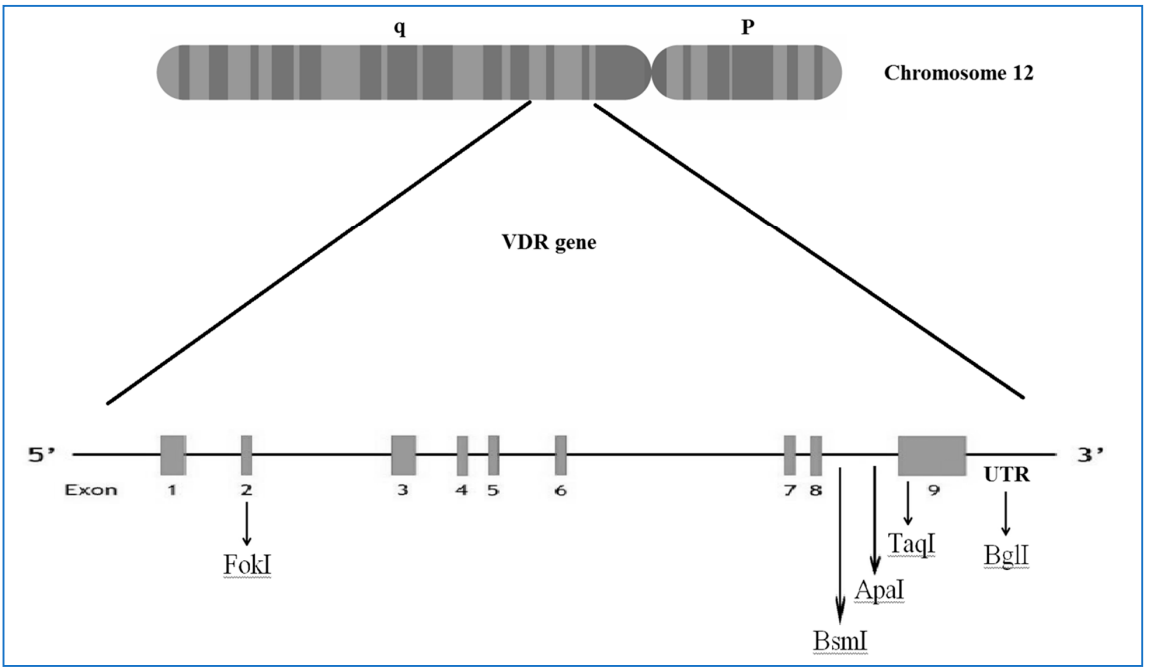

Figure 3. The location of vitamin D receptor $(V D R)$ polymorphisms reported in the meta-analysis. 
The meta-analysis has several limitations and strengths. Limitations include the presence of fewer published reports on this topic hindering the performance of any metaregression analysis, studies with small sample sizes, and clinical and statistical heterogeneity between the studies. Some studies included in the meta-analysis did not match cases with controls, used genotyping methods different from other studies, and had controls with a deviation of the HWE. It also needs mentioning that we could not conduct any analysis to adjust the effect of multiple testing or multiplicity within the included studies. Despite the limitations, this review demonstrates several strengths in the form of the lack of publication bias, the suitable quality of all the included studies, and the use a population-based source for recruiting controls in all the studies. More studies on larger sample sizes and different ethnicities will help to explore the influence of different $V D R$ polymorphisms on the risk of dental caries.

\section{Conclusions}

Out of the six VDR polymorphisms explored in this meta-analysis, an association was only observed between the FokI (rs10735810) polymorphism and the risk of dental caries, with the $f$ allele and ff genotype demonstrating a protective role in the occurrence of dental caries.

Author Contributions: Conceptualization, M.S.; methodology, M.S. and S.K.T.; validation, M.S. and S.K.T.; formal analysis, M.S.; investigation, M.S.; resources, M.S.; data curation, M.S.; writingoriginal draft preparation, S.K.T.; writing-review and editing, M.S., A.G., M.G., R.S., A.K. and N.N.; visualization, S.K.T.; supervision, A.G., M.G. and R.S.; project administration, M.S. All authors have read and agreed to the published version of the manuscript.

Funding: This research received no external funding.

Institutional Review Board Statement: Not applicable.

Informed Consent Statement: Not applicable.

Acknowledgments: Santosh Kumar Tadakamadla acknowledges the support of NHMRC Early Career Fellowship.

Conflicts of Interest: The authors declare no conflict of interest.

\section{References}

1. Petersen, P.E. The World Oral Health Report 2003: Continuous improvement of oral health in the 21st century-The approach of the WHO Global Oral Health Programme. Community Dent. Oral Epidemiol. 2003, 31, 3-24. [CrossRef] [PubMed]

2. Petersen, P.E.; Bourgeois, D.; Ogawa, H.; Estupinan-Day, S.; Ndiaye, C. The global burden of oral diseases and risks to oral health Bull. World Health Organ. 2005, 83, 661-669.

3. Selwitz, R.; Ismail, A.; Pitts, N. Dental caries. Lancet 2007, 369, 51-59. [CrossRef]

4. Collaborators, G.O.D.; Bernabe, E.; Marcenes, W.; Hernandez, C.; Bailey, J.; Abreu, L.; Alipour, V.; Amini, S.; Arabloo, J.; Arefi, Z. Global, regional, and national levels and trends in burden of oral conditions from 1990 to 2017: A systematic analysis for the global burden of disease 2017 study. J. Dent. Res. 2020, 99, 362-373. [CrossRef] [PubMed]

5. Vieira, A.R.; Modesto, A.; Marazita, M.L. Caries: Review of human genetics research. Caries Res. 2014, 48, 491-506. [CrossRef] [PubMed]

6. Haworth, S.; Shungin, D.; Van Der Tas, J.T.; Vucic, S.; Medina-Gomez, C.; Yakimov, V.; Feenstra, B.; Shaffer, J.R.; Lee, M.K.; Standl, M. Consortium-based genome-wide meta-analysis for childhood dental caries traits. Hum. Mol. Genet. 2018, $27,3113-3127$. [CrossRef] [PubMed]

7. Shaffer, J.; Wang, X.; Feingold, E.; Lee, M.; Begum, F.; Weeks, D.; Cuenco, K.; Barmada, M.; Wendell, S.; Crosslin, D. Genome-wide association scan for childhood caries implicates novel genes. J. Dent. Res. 2011, 90, 1457-1462. [CrossRef] [PubMed]

8. Chapple, I.L.; Bouchard, P.; Cagetti, M.G.; Campus, G.; Carra, M.C.; Cocco, F.; Nibali, L.; Hujoel, P.; Laine, M.L.; Lingström, P. Interaction of lifestyle, behaviour or systemic diseases with dental caries and periodontal diseases: Consensus report of group 2 of the joint EFP/ORCA workshop on the boundaries between caries and periodontal diseases. J. Clin. Periodontol. 2017, 44, S39-S51. [CrossRef]

9. Wang, X.; Shaffer, J.; Weyant, R.; Cuenco, K.; DeSensi, R.; Crout, R.; McNeil, D.; Marazita, M. Genes and their effects on dental caries may differ between primary and permanent dentitions. Caries Res. 2010, 44, 277-284. [CrossRef]

10. Wendell, S.; Wang, X.; Brown, M.; Cooper, M.; DeSensi, R.; Weyant, R.; Crout, R.; McNeil, D.; Marazita, M. Taste genes associated with dental caries. J. Dent. Res. 2010, 89, 1198-1202. [CrossRef] 
11. Randev, S.; Kumar, P.; Guglani, V. Vitamin D supplementation in childhood-A review of guidelines. Indian J. Pediatrics 2018, 85, 194-201. [CrossRef] [PubMed]

12. Chhonkar, A.; Gupta, A.; Arya, V. Comparison of vitamin D level of children with severe early childhood caries and children with no caries. Int. J. Clin. Pediatric Dent. 2018, 11, 199. [CrossRef]

13. Deane, S.; Schroth, R.J.; Sharma, A.; Rodd, C. Combined deficiencies of 25-hydroxyvitamin D and anemia in preschool children with severe early childhood caries: A case-control study. Paediatr. Child Health 2018, 23, e40-e45. [CrossRef] [PubMed]

14. Gyll, J.; Ridell, K.; Öhlund, I.; Åkeson, P.K.; Johansson, I.; Holgerson, P.L. Vitamin D status and dental caries in healthy Swedish children. Nutr. J. 2018, 17, 1-10. [CrossRef] [PubMed]

15. Kim, I.-J.; Lee, H.-S.; Ju, H.-J.; Na, J.-Y.; Oh, H.-W. A cross-sectional study on the association between vitamin D levels and caries in the permanent dentition of Korean children. BMC Oral Health 2018, 18, 1-6. [CrossRef]

16. Sutton, A.L.; MacDonald, P.N. Vitamin D: More than a "bone-a-fide" hormone. Mol. Endocrinol. 2003, 17, 777-791. [CrossRef]

17. Valdivielso, J.M.; Fernandez, E. Vitamin D receptor polymorphisms and diseases. Clin. Chim. Acta 2006, 371, 1-12. [CrossRef] [PubMed]

18. Uitterlinden, A.G.; Fang, Y.; Van Meurs, J.B.; Pols, H.A.; Van Leeuwen, J.P. Genetics and biology of vitamin D receptor polymorphisms. Gene 2004, 338, 143-156. [CrossRef]

19. Uitterlinden, A.G.; Fang, Y.; van Meurs, J.B.; van Leeuwen, H.; Pols, H.A. Vitamin D receptor gene polymorphisms in relation to Vitamin D related disease states. J. Steroid Biochem. Mol. Biol. 2004, 89, 187-193. [CrossRef]

20. Hujoel, P.P. Vitamin D and dental caries in controlled clinical trials: Systematic review and meta-analysis. Nutr. Rev. 2013, 71, 88-97. [CrossRef] [PubMed]

21. Kong, Y.-Y.; Zheng, J.-M.; Zhang, W.-J.; Jiang, Q.-Z.; Yang, X.-C.; Yu, M.; Zeng, S.-J. The relationship between vitamin D receptor gene polymorphism and deciduous tooth decay in Chinese children. BMC Oral Health 2017, 17, 1-6. [CrossRef] [PubMed]

22. Patir, A.; Seymen, F.; Yildirim, M.; Deeley, K.; Cooper, M.; Marazita, M.; Vieira, A. Enamel formation genes are associated with high caries experience in Turkish children. Caries Res. 2008, 42, 394-400. [CrossRef]

23. Sharifi, R.; Jahedi, S.; Mozaffari, H.R.; Imani, M.M.; Sadeghi, M.; Golshah, A.; Moradpoor, H.; Safaei, M. Association of LTF, ENAM, and AMELX polymorphisms with dental caries susceptibility: A meta-analysis. BMC Oral Health 2020, 20, 1-11. [CrossRef]

24. Triantos, C.; Aggeletopoulou, I.; Kalafateli, M.; Spantidea, P.I.; Vourli, G.; Diamantopoulou, G.; Tapratzi, D.; Michalaki, M.; Manolakopoulos, S.; Gogos, C. Prognostic significance of vitamin D receptor (VDR) gene polymorphisms in liver cirrhosis. Sci. Rep. 2018, 8, 1-14. [CrossRef]

25. Zhang, P.; Li, Q.; Qi, J.; Lv, Q.; Zheng, X.; Wu, X.; Gu, J. Association between vitamin D receptor gene polymorphism and ankylosing spondylitis in Han Chinese. Int. J. Rheum. Dis. 2017, 20, 1510-1516. [CrossRef]

26. Moher, D.; Liberati, A.; Tetzlaff, J.; Altman, D.G. Preferred reporting items for systematic reviews and meta-analyses: The PRISMA statement. Int. J. Surg. 2010, 8, 336-341. [CrossRef]

27. Higgins, J.P.; Thompson, S.G. Quantifying heterogeneity in a meta-analysis. Stat. Med. 2002, 21, 1539-1558. [CrossRef] [PubMed]

28. Higgins, J.P.; Thompson, S.G.; Deeks, J.J.; Altman, D.G. Measuring inconsistency in meta-analyses. BMJ 2003, 327, 557-560. [CrossRef]

29. Machiela, M.J.; Chanock, S.J. LDlink: A web-based application for exploring population-specific haplotype structure and linking correlated alleles of possible functional variants. Bioinformatics 2015, 31, 3555-3557. [CrossRef] [PubMed]

30. Aribam, V.G.; Aswath, N.; Ramanathan, A. Single-nucleotide polymorphism in Vitamin D receptor gene and its association with dental caries in children. J. Indian Soc. Pedod. Prev. Dent. 2020, 38, 8.

31. Barbosa, M.C.; Lima, D.C.; Reis, C.L.; Reis, A.L.; Rigo, D., Jr.; Segato, R.A.; Storrer, C.L.; Küchler, E.C.; de Oliveira, D.S. Vitamin D receptor FokI and BgII genetic polymorphisms, dental caries, and gingivitis. Int. J. Paediatr. Dent. 2020, 30, 642-649. [CrossRef]

32. Cogulu, D.; Onay, H.; Ozdemir, Y.; Aslan, G.I.; Ozkinay, F.; Eronat, C. The role of vitamin D receptor polymorphisms on dental caries. J. Clin. Pediatric Dent. 2016, 40, 211-214. [CrossRef] [PubMed]

33. Fatturi, A.L.; Menoncin, B.L.; Reyes, M.T.; Meger, M.; Scariot, R.; Brancher, J.A.; Küchler, E.C.; Feltrin-Souza, J. The relationship between molar incisor hypomineralization, dental caries, socioeconomic factors, and polymorphisms in the vitamin $\mathrm{D}$ receptor gene: A population-based study. Clin. Oral Investig. 2020, 24, 3971-3980. [CrossRef]

34. Holla, L.I.; Linhartova, P.B.; Kastovsky, J.; Bartosova, M.; Musilova, K.; Kukla, L.; Kukletova, M. Vitamin D receptor TaqI gene polymorphism and dental caries in Czech children. Caries Res. 2017, 51, 7-11. [CrossRef] [PubMed]

35. Madalena, I.R.; Xavier, T.A.; Cruz, G.V.; Brancher, J.A.; da Silva, L.A.B.; Paza, A.O.; Segato, R.A.B.; Küchler, E.C. Evaluation of vitamin D receptor genetic polymorphisms with dental caries and developmental defects of enamel in Brazilian children. Pediatric Dent. J. 2020, 30, 161-166. [CrossRef]

36. Qin, X.; Shao, L.; Zhang, L.; Ma, L.; Xiong, S. Investigation of Interaction between Vitamin D Receptor Gene Polymorphisms and Environmental Factors in Early Childhood Caries in Chinese Children. Biomed Res. Int. 2019, 2019. [CrossRef] [PubMed]

37. Yu, M.; Jiang, Q.-Z.; Sun, Z.-Y.; Kong, Y.-Y.; Chen, Z. Association between single nucleotide polymorphisms in vitamin D receptor gene polymorphisms and permanent tooth caries susceptibility to permanent tooth caries in Chinese adolescent. Biomed Res. Int. 2017, 2017. [CrossRef]

38. Ryan, J.W.; Anderson, P.H.; Morris, H.A. Pleiotropic activities of vitamin D receptors-adequate activation for multiple health outcomes. Clin. Biochem. Rev. 2015, 36, 53.

39. Dusso, A.S.; Brown, A.J. Mechanism of vitamin D action and its regulation. Am. J. Kidney Dis. 1998, 32, S13-S24. [CrossRef] 
40. Amano, Y.; Komiyama, K.; Makishima, M. Vitamin D and periodontal disease. J. Oral Sci. 2009, 51, 11-20. [CrossRef]

41. Demay, M.B. Mechanism of vitamin D receptor action. Ann. N. Y. Acad. Sci. 2006, 1068, 204-213. [CrossRef] [PubMed]

42. Cooper, G.S.; Umbach, D.M. Are vitamin D receptor polymorphisms associated with bone mineral density? A meta-analysis. J. Bone Miner. Res. 1996, 11, 1841-1849. [CrossRef] [PubMed]

43. Barry, E.L.; Peacock, J.L.; Rees, J.R.; Bostick, R.M.; Robertson, D.J.; Bresalier, R.S.; Baron, J.A. Vitamin D receptor genotype, vitamin D3 supplementation, and risk of colorectal adenomas: A randomized clinical trial. JAMA Oncol. 2017, 3, 628-635. [CrossRef] [PubMed]

44. Karasneh, J.A.; Ababneh, K.T.; Taha, A.H.; Al-Abbadi, M.S.; Marzouka, N.A.d.S.; Jaradat, S.M.; Thornhill, M.H. Association of vitamin D receptor gene polymorphisms with chronic and aggressive periodontitis in Jordanian patients. Eur. J. Oral Sci. 2013, 121, 551-558. [CrossRef]

45. Sarkissyan, M.; Wu, Y.; Chen, Z.; Mishra, D.K.; Sarkissyan, S.; Giannikopoulos, I.; Vadgama, J.V. Vitamin D receptor FokI gene polymorphisms may be associated with colorectal cancer among African American and Hispanic participants. Cancer 2014, 120, 1387-1393. [CrossRef]

46. Vanessa, O.; Asani, F.F.; Jeffery, T.J.; Saccone, D.S.; Bornman, L. Vitamin D receptor gene expression and function in a South African population: Ethnicity, vitamin D and Fok I. PLoS ONE 2013, 8, e67663.

47. Nelson, D.; Vord, P.V.; Wooley, P. Polymorphism in the vitamin D receptor gene and bone mass in African-American and white mothers and children: A preliminary report. Ann. Rheum. Dis. 2000, 59, 626-630. [CrossRef] [PubMed]

48. Tanaka, K.; Miyake, Y.; Hanioka, T.; Arakawa, M. VDR gene polymorphisms, interaction with smoking and risk of periodontal disease in Japanese women: The Kyushu Okinawa maternal and child health study. Scand. J. Immunol. 2013, 78, 371-377. [CrossRef] 